\title{
ON TORSION-FREE ABELIAN GROUPS AND LIE ALGEBRAS
}

\section{RICHARD BLOCK}

It is known that many of the classes of simple Lie algebras of prime characteristic of nonclassical type have simple infinite-dimensional analogues of characteristic zero (see, for example, $[4$, p. 518]). We consider here analogues of those algebras which are defined by a modification of the definition of a group algebra. Thus we consider analogues of the Zassenhaus algebras as generalized by Albert and Frank in [1].

The algebras considered are defined as follows. Let $G$ be a nonzero abelian group, $F$ a field, $g$ an additive mapping from $G$ to $F$, and $f$ an alternate biadditive mapping from $G \times G$ to $F$. We index a basis of an algebra over $F$ by $G$, denoting by $u_{\alpha}$ the basis element corresponding to $\alpha$ in $G$, and define multiplication by

$$
u_{\alpha} u_{\beta}=\{f(\alpha, \beta)+g(\alpha-\beta)\} u_{\alpha+\beta} .
$$

We designate this algebra by $L(G, g, f)$. We shall determine necessary and sufficient conditions on $f$ and $g$ for the algebra $L(G, g, f)$ to be a simple Lie algebra. We shall then consider the case in which $L(G, g, f)$ is a simple Lie algebra of characteristic zero. This will be seen to imply that $G$ is torsion-free. The derivations and locally algebraic derivations of $L(G, g, f)$ will be determined in this case. Using these, we shall show that any one of these simple Lie algebras $L(G, g, f)$ of characteristic zero determines the group $G$ up to isomorphism and determines the mappings $g$ and $f$ up to a scalar multiple.

Our proof of the simplicity of $L(G, g, f)$ and determination of the derivations of $L(G, g, f)$ are also valid when $F$ has prime characteristic $p$ and $G$ is an elementary abelian $p$-group. However in that case our method for showing that $L(G, g, f)$ essentially determines $G, g$ and $f$ cannot be used-indeed, Ree showed in [4] that all Zassenhaus algebras of dimension $p^{n}$ over $F$ are isomorphic.

When the torsion-free abelian group $G$ has rank one, the simple algebra $L(G, g, f)$ over $F$, of characteristic zero, is isomorphic to the algebra of derivations of the group algebra of $G$ over $F$. Thus the group algebra of a torsion-free abelian group of rank one determines the group. However this is a special case of a result that follows from Higman's determination of the units of group algebras in [2].

Presented to the Society January 29, 1958, under the title of Relations between torsion-free abelian groups and certain Lie algebras; received by the editors March 10 , 1958. 
2. Simplicity. It was shown by Albert and Frank in $[1$, p. 132 $]$ that when $g(\alpha)=0$ for all $\alpha$ in $G$ then the algebra $L(G, g, f)$ is a Lie algebra and contains the one-dimensional ideal spanned by $u_{0}$, and that when $g$ does not vanish identically then $L(G, g, f)$ is a Lie algebra if and only if there is an (additive) mapping $h$ on $G$ to $F$ such that

$$
f(\alpha, \beta)=g(\alpha) h(\beta)-g(\beta) h(\alpha)
$$

for all $\alpha$ and $\beta$ in $G$.

Now suppose that $g$ does not vanish identically and that an $h$ is given such that (2) holds. We seek to determine conditions under which $L(G, g, f)$ will be simple. If $g(\gamma)=h(\gamma)=0$ for some nonzero $\gamma$ then in case $\gamma$ has finite order $q$, all elements of the form

$$
\sum_{i=0}^{q-1} u_{\alpha+i \gamma}
$$

span a proper ideal, while if $\gamma$ has infinite order, all elements of the form $u_{\alpha}+u_{\alpha+\gamma}$ span a proper ideal. Also if there is an element $\delta$ in $G$ such that $g(\delta)=0$ and $h(\delta)=2$ (in particular for characteristic two, if $\delta=0)$ then all $u_{\alpha}$ with $\alpha \neq-\delta$ span a proper ideal of $L(G, g, f)$.

We therefore suppose that there is no nonzero $\delta$ in $G$ such that $g(\delta)=0$ and $h(\delta)=0$ or 2 , and that the characteristic is not two. Thus there is no $\delta$ in $G$ such that $g(\delta)=0$ and $h(\delta)= \pm 1$. Also, by (2), either the kernel of $g$ is zero or $f$ is nonsingular.

The length $\lambda(x)$ of an element $x=\sum_{\beta} a_{\beta} u_{\beta}$ of $L(G, g, f)$ denotes the number of nonzero coefficients $a_{\beta}$ in $x$, and $\beta$ is said to be $x$-admissible if $a_{\beta} \neq 0$.

Now, under the above conditions, let $M$ be a nonzero ideal of $L(G, g, f), x$ a nonzero element of $M$ of minimal length, and suppose $\lambda(x)>1$. If $g(\alpha)=0$ for some $x$-admissible $\alpha$, then with $\gamma$ such that $g(\gamma) \neq 0$, we have $\lambda\left(x u_{\gamma}\right) \leqq \lambda(x), f(\alpha, \gamma)+g(\alpha-\gamma)=-g(\gamma)[h(\alpha)+1] \neq 0$ and $\alpha+\gamma$ is $\left(x u_{\gamma}\right)$-admissible. Hence we may assume that $g(\beta) \neq 0$ for some $x$-admissible $\beta$. Now if $g(\alpha)=0$ for some $x$-admissible $\alpha$ then $x u_{\alpha} \neq 0$ since $f(\beta, \alpha)+g(\beta-\alpha)=g(\beta)[h(\alpha)+1] \neq 0$, but $\lambda\left(x u_{\alpha}\right)<\lambda(x)$, a contradiction. Hence for every $x$-admissible $\beta, g(\beta) \neq 0$. Take an $x$-admissible $\alpha$ and let $y=x u_{-\alpha}$. Then 0 is $y$-admissible since $f(\alpha,-\alpha)$ $+g(\alpha)-g(-\alpha)=2 g(\alpha) \neq 0$, so $y \neq 0$ and $\lambda(y)=\lambda(x)$. Thus $\beta-\alpha$ is $y$ admissible for every $x$-admissible $\beta$, and since 0 is $y$-admissible, $g(\beta-\alpha)=0$ for every $x$-admissible $\beta$. Now if $\alpha$ and $\beta$ are $x$-admissible and $\alpha \neq \beta$, then since $\lambda\left(x u_{\beta}\right)<\lambda(x)$, we have $x u_{\beta}=0,0=f(\alpha, \beta)$ $+g(\alpha-\beta)=g(\alpha)[h(\beta-\alpha)]$, so $g(\alpha-\beta)=h(\alpha-\beta)=0$ and $\alpha=\beta$, a contradiction.

Hence $\lambda(x)=1$ and $M$ contains some $u_{\alpha}$. If $g(\alpha)=0$ then for $\beta$ such 
that $g(\beta) \neq 0, M$ contains $u_{\alpha} u_{\beta}=-g(\beta)[h(\alpha)+1] u_{\alpha+\beta} \neq 0$, so $M$ contains a $u_{\gamma}$ with $g(\gamma) \neq 0$. Now if $\beta$ is such that $g(\beta)=0$ then $M$ contains $u_{\beta}$, since $u_{\gamma} u_{-\gamma+\beta}=g(\gamma)[h(\beta)+2] u_{\beta} \neq 0$. In particular $M$ contains $u_{0}$ and so also any $u_{\alpha}$ with $g(\alpha) \neq 0$, since $u_{0} u_{\alpha}=-g(\alpha) u_{\alpha} \neq 0$. Therefore $M=L$, and we have proved the following theorem.

Theorem 1. The algebra $L(G, g, f)$ defined by (1) is a simple Lie algebra if and only if the characteristic is not two, $g$ does not vanish identically and there is an additive mapping $h$ from $G$ to $F$ for which (2) holds and for which there is no nonzero $\delta$ in $G$ with $g(\delta)=0$ and $h(\delta)=0$ or 2.

From the conditions of this theorem on the mappings $g$ and $h$, the following result may be proved easily.

Corollary 1. Suppose that $F$ is a field of characteristic 0 , of degree d (finite or infinite) over the rationals. Then if $L(G, g, f)$ is a simple Lie algebra over $F$, the group $G$ must be torsion-free. Let $G$ be a given torsionfree abelian group and let $r$ be the rank (the maximum number of linearly independent elements) of $G$. Then there exists a simple Lie algebra $L(G, g, f)$ over $F$ if and only if $2 d \geqq r$, when $G$ is not divisible, or $2 d$ $\geqq r+1$, when $G$ is divisible.

Similar statements hold when $F$ has prime characteristic $p$. In that case $G$ must be an elementary $p$-group in order for $L(G, g, f)$ to be a simple Lie algebra. The Lie algebras $L(G, g, f)$ were shown to be simple when $g$ is an isomorphism by Albert and Frank in [1, p. 138]. In the finite dimensional case the simple Lie algebras $L(G, g, f)$ may be shown to be the same up to isomorphism as the $p^{n}$-dimensional simple Lie algebras considered by Jennings and Ree in [3].

3. The algebra of derivations of $L(G, g, f)$. We shall henceforth assume that any algebra $L(G, g, f)$ considered is a simple Lie algebra, and in particular that a mapping $h$ satisfying the conditions of Theorem 1 is given.

Now suppose that $D$ is a derivation of $L(G, g, f)$ and let $c(\alpha, \gamma)$ be the coefficient of $u_{\alpha+\gamma}$ in $u_{\alpha} D$, that is,

$$
D: u_{\alpha} \rightarrow \sum_{\gamma \in G} c(\alpha, \gamma) u_{\alpha+\gamma}=\sum_{\gamma \in G} c(\alpha,-\alpha+\gamma) u_{\gamma}
$$

the sums being finite of course. Since $D$ is a derivation, $\left(u_{\gamma} u_{\epsilon}\right) D$ $=\left(u_{\gamma} D\right) u_{\epsilon}+u_{\gamma}\left(u_{\epsilon} D\right)$, that is, with $\phi(\alpha, \beta)$ denoting $f(\alpha, \beta)+g(\alpha-\beta)$,

$$
\begin{aligned}
\sum_{\zeta \in G}\{\phi(\gamma, \epsilon) c(\gamma+\epsilon,-\gamma-\epsilon & +\zeta)-\phi(\zeta-\epsilon, \epsilon) c(\gamma,-\gamma-\epsilon+\zeta) \\
& -\phi(\gamma,-\gamma+\zeta) c(\epsilon,-\gamma-\epsilon+\zeta)\} u_{\zeta}=0
\end{aligned}
$$


for all $\gamma$ and $\epsilon$ in $G$. Taking $\zeta=\gamma+\epsilon+\theta$ this gives

$$
\begin{aligned}
{[f(\gamma, \epsilon)+g(\gamma-\epsilon)] c(\gamma+\epsilon, \theta) } & =[f(\gamma+\theta, \epsilon)+g(\gamma-\epsilon+\theta)] c(\gamma, \theta) \\
& +[f(\gamma, \epsilon+\theta)+g(\gamma-\epsilon-\theta)] c(\epsilon, \theta)
\end{aligned}
$$

for all $\gamma, \epsilon$ and $\theta$ in $G$.

Lemma 1. If $\theta \neq 0$ then

$$
[f(\beta, \theta)+g(\beta-\theta)] c(\alpha, \theta)=[f(\alpha, \theta)+g(\alpha-\theta)] c(\beta, \theta)
$$

for any $\alpha$ and $\beta$ in $G$.

In this proof we denote $c(\gamma, \theta)$ by $c_{\gamma}$ for any $\gamma$. The proof is divided into two cases.

CASE I. $g(\theta) \neq 0$. Taking $\gamma=\alpha$ and $\epsilon=0$ in (3), we get $0=g(\theta) c_{\alpha}$ $+[f(\alpha, \theta)+g(\alpha-\theta)] c_{0}$, i.e.,

$$
c_{\alpha}=[f(\alpha, \theta)+g(\alpha-\theta)][-g(\theta)]^{-1} c_{0}
$$

which, together with the similar result for $\beta$, gives (4).

CASE II. $g(\theta)=0, \theta \neq 0$. If $g(\alpha)=g(\beta)=0$ then both sides of (4) vanish, so we may assume that, say, $g(\alpha) \neq 0$. With $\gamma=\alpha$ and $\epsilon=0$, (3) gives $g(\alpha)[h(\theta)+1] c_{0}=0$, i.e., $c_{0}=0$. Now let $\zeta$ and $\eta$ be any nonzero elements of $G$ such that $g(\zeta) \neq 0$ and $g(\eta)=0$. Then (3) with $\gamma=\zeta$ and $\epsilon=-\zeta$ gives $0=g(\zeta)[h(\theta)+2]\left\{c_{\zeta}+c_{-\zeta}\right\}$, i.e.,

$$
c_{-\zeta}=-c_{\zeta} \text {. }
$$

Also by $(3), g(\zeta)[h(\eta)+1] c_{\zeta+\eta}=g(\zeta)[h(\eta)+1] c_{\zeta}+g(\zeta)[h(\eta+\theta)+1] c_{\eta}$, i.e.,

$$
c_{\zeta+\eta}=c_{\zeta}+[h(\eta)+1]^{-1}[h(\eta+\theta)+1] c_{\eta},
$$

while with $\gamma=-\zeta$ and $\epsilon=\zeta+\eta$, (3) gives

$$
-g(\zeta)[h(\eta)+2] c_{\eta}=-g(\zeta)[h(\eta+\theta)+2]\left\{c_{-\zeta}+c_{\zeta+\eta}\right\}
$$

i.e.,

$$
c_{\zeta+n}=-c_{-\zeta}+[h(\eta+\theta)+2]^{-1}[h(\eta)+2] c_{\eta} .
$$

Hence $[h(\eta+\theta)+2][h(\eta+\theta)+1] c_{\eta}=[h(\eta)+1][h(\eta)+2] c_{\eta}$, so that $h(\theta)[h(2 \eta+\theta)+3] c_{\eta}=0$. Thus $c_{\eta}=0$ and $c_{\zeta+\eta}=c_{\zeta}$ if $h(2 \eta+\theta) \neq-3$ while if $h(2 \eta+\theta)=-3$ then, since $h(4 \eta+\theta) \neq-3$ and $h(-2 \eta+\theta)$ $\neq-3$, we have $c_{\zeta+\eta}=c_{\zeta-\eta+2 \eta}=c_{\zeta-\eta}=c_{\zeta}$, and again $c_{\eta}=0$. It follows that

$$
c_{\alpha+\beta+\theta}=c_{\alpha+\beta}, \quad c_{\beta+\theta}=c_{\beta} .
$$

Now with $\gamma=\alpha$ and $\epsilon=\beta+\theta$ in (3) we have 


$$
\begin{aligned}
& {[f(\alpha, \beta+\theta)+g(\alpha-\beta)] c_{\alpha+\beta}} \\
& \quad=[f(\alpha+\theta, \beta+\theta)+g(\alpha-\beta)] c_{\alpha}+[f(\alpha, \beta+2 \theta)+g(\alpha-\beta)] c_{\beta} .
\end{aligned}
$$

Subtracting from this (3) with $\gamma=\alpha$ and $\epsilon=\beta$ we get $f(\alpha, \theta) c_{\alpha+\beta}$ $=f(\alpha, \theta)\left\{c_{\alpha}+c_{\beta}\right\}$, so that $c_{\alpha+\beta}=c_{\alpha}+c_{\beta}$. Now (3) with $\gamma=\alpha$ and $\epsilon=\beta$ gives

$$
f(\beta, \theta) c_{\alpha}=f(\alpha, \theta) c_{\beta} .
$$

Multiplying both sides of this by $[h(\theta)]^{-1}[h(\theta)+1]$, we get (4) for this case also, which proves the lemma.

Lemma 2. The derivation $D$ differs by an inner derivation $D^{\prime}$ from a derivation for which the coefficients $c(\alpha, \theta)$ vanish for all nonzero $\theta$.

Indeed for any nonzero $\theta$ we may take an $\alpha$ such that $f(\alpha, \theta)$ $+g(\alpha-\theta) \neq 0$ and set $k_{\theta}=[f(\alpha, \theta)+g(\alpha-\theta)]^{-1} c(\alpha, \theta)$. By Lemma 1 , $k_{\theta}$ is well defined. Thus if $g(\theta) \neq 0$ then $k_{\theta}=-[g(\theta)]^{-1} c(0, \theta)$, and since $c(0, \theta) \neq 0$ for only finitely many $\theta$, there are only finitely many $\theta$ such that $g(\theta) \neq 0$ and $k_{\theta} \neq 0$. Similarly, taking $\alpha$ such that $g(\alpha) \neq 0$, we find that there are also only finitely many $\theta$ such that $g(\theta)=0$ and $k_{\theta} \neq 0$. Therefore we may consider the right multiplication by $\sum_{\theta \neq 0} k_{\theta} u_{\theta}$. Taking this to be $D^{\prime}$, and noting that by Lemma 1 if $f(\alpha, \theta)+g(\alpha-\theta)=0$ then $c(\alpha, \theta)=0$ (for nonzero $\theta$ ), we see that the lemma holds.

Now let $d$ be any additive function of $G$ to $F$. Then it is easy to see that the linear transformation $D_{d}$ determined by the mapping of the basis elements

$$
u_{\alpha} \rightarrow d(\alpha) u_{\alpha}
$$

is a derivation of $L(G, g, f)$.

TheOREм 2. The algebra of derivations of the simple Lie algebra $L(G, g, f)$ is spanned by the inner derivations together with all the derivations $D_{d}$.

What remains to be proved is that

$$
c_{\alpha+\beta}=c_{\alpha}+c_{\beta}
$$

for any $\alpha$ and $\beta$, where, for any $\gamma, c_{\gamma}$ denotes $c(\gamma, 0)$. If $f(\alpha, \beta)$ $+g(\alpha-\beta) \neq 0$, (5) follows directly from (3) with $\theta=0$. But if $f(\alpha, \beta)$ $+g(\alpha-\beta)=0$ then we may pick a $\gamma$ such that the expressions

$$
\begin{gathered}
f(\alpha+\beta, \gamma)+g(\alpha+\beta-\gamma), \quad f(\alpha, \beta+\gamma)+g(\alpha-\beta-\gamma), \\
f(\beta, \gamma)+g(\beta-\gamma)
\end{gathered}
$$


are all nonzero, for if $g(\alpha)=0$ (so that $g(\beta)=0$ also) then any $\gamma$ for which $g(\gamma) \neq 0$ will do, while if $g(\alpha) \neq 0$ we may take $\gamma=2 \beta$ unless $\alpha=\beta$. When $g(\alpha) \neq 0$ and $\alpha=\beta$ (and the characteristic is not 3) we may take $\gamma=-\alpha$. Now with such a $\gamma$ we have

$$
c_{\alpha+\beta}+c_{\gamma}=c_{\alpha+\beta+\gamma}=c_{\alpha}+c_{\beta+\gamma}=c_{\alpha}+c_{\beta}+c_{\gamma}
$$

(when the characteristic is 3 and $\alpha=\beta$ one argues that $c_{2 \alpha}=c_{-\alpha}$ $=-c_{\alpha}=2 c_{\alpha}$ ). Thus (5) holds and the theorem is proved.

Noting that the derivation sending $u_{\alpha}$ to $g(\alpha) u_{\alpha}$ is inner, we obtain the following result.

COROllary 2. If $G$ has finite rank $n$ then the algebra of outer derivations of $L(G, g, f)$ is an abelian Lie algebra of dimension $n-1$.

4. Criteria for isomorphism. Henceforth we shall restrict our consideration to simple Lie algebras $L(G, g, f)$ of characteristic zero, so that $G$ must be torsion-free.

A derivation $D$ of an algebra $L$ is locally algebraic if and only if it is true that for every $x$ in $L$ the set $\left\{x D^{i}: i=1,2, \cdots\right\}$ lies in a finite-dimensional subspace (depending on $x$ ) of $L$.

Lemma 3. The only locally algebraic derivations of $L(G, g, f)$ are the derivations $D_{d}$.

The derivations $D_{d}$ are obviously locally algebraic. Now suppose that $D$ is a locally algebraic derivation. By Theorem $2, D=R_{y}+D_{d}$, where $R_{y}$ is the right multiplication by $y=\sum_{\gamma} a_{\gamma} u_{\gamma}$, for some $y$ and $d$. Suppose that some nonzero $\gamma$ is $y$-admissible. We may simply order $G$ in such a way that this $\gamma>0$. Call $u_{\epsilon}$ the leading term in an element $z$ of $L(G, g, f)$ if $\epsilon$ is the greatest $z$-admissible element of $G$, and let $u_{\alpha}$ be the leading term in $y$. Thus $\alpha>0$. We shall find a $\beta$ such that the leading term in $\left(u_{\beta}\right) D^{i}$ is $u_{\beta+i \alpha}$, contradicting the assumption that $D$ is locally algebraic. Indeed if $g(\alpha)=0$ we may take $\beta$ to be any positive element of $G$ with $g(\beta) \neq 0$, since then the coefficient of $u_{\beta+i \alpha}$ in $\left(u_{\beta}\right) D^{i}$ is $[g(\beta)]^{i}[h(\alpha)+1]^{i} \neq 0$, while if $g(\alpha) \neq 0$ we may take $\beta$ to be $2 \alpha$. Thus $y$ must be a scalar multiple of $u_{0}$, and the lemma is proved.

Since for any distinct elements $\alpha$ and $\beta$ there is an additive mapping $d$ of $G$ to $F$ such that $d(\alpha) \neq d(\beta)$, we have the following result.

Lemma 4 . The only elements of $L(G, g, f)$ which are characteristic vectors for all locally algebraic derivations are the scalar multiples of all the elements $u_{\alpha}$.

Now suppose that $\sigma$ is an isomorphism of one algebra $L(G, g, f)$ onto another, $L\left(G^{\prime}, g^{\prime}, f^{\prime}\right)$. We shall determine the relations between 
$G, g$ and $f$ on the one hand, and $G^{\prime}, g^{\prime}$ and $f^{\prime}$ on the other. It follows from Lemma 4 that for every $\alpha$ in $G$ there is an element $\alpha^{\sigma}$ in $G^{\prime}$ such that

$$
\left(u_{\alpha}\right) \sigma=c l_{\alpha} u_{\alpha^{\sigma}}
$$

where $l_{\alpha}$ is a nonzero scalar (depending on $\alpha$ and $\sigma$ ) and $c$ is a fixed scalar chosen so that $l_{0}=1$. The induced mapping $\sigma: \alpha \rightarrow \alpha^{\sigma}$ of $G$ into $G^{\prime}$ is one-to-one and onto. Since $\left(u_{\gamma} u_{\epsilon}\right) \sigma=\left[\left(u_{\gamma}\right) \sigma\right]\left[\left(u_{\epsilon}\right) \sigma\right]$,

$$
\begin{aligned}
c l_{\gamma+\epsilon}[f(\gamma, \epsilon)+g(\gamma-\epsilon)] u_{(\gamma+\epsilon)^{\sigma}} & =c^{2} l_{\gamma} l_{\epsilon}\left[f^{\prime}\left(\gamma^{\sigma}, \epsilon^{\sigma}\right)+g^{\prime}\left(\gamma^{\sigma}-\epsilon^{\sigma}\right)\right] u_{\gamma^{\sigma}+\epsilon^{\sigma}}
\end{aligned}
$$

for all $\gamma$ and $\boldsymbol{\epsilon}$ in $G$. Hence if $f(\gamma, \epsilon)+g(\gamma-\epsilon) \neq 0$ then $(\gamma+\epsilon)^{\sigma}=\gamma^{\sigma}+\epsilon^{\sigma}$, so that, exactly as in the final part of the proof of Theorem 2, $\sigma$ is always additive and therefore is an isomorphism of $G$ onto $G^{\prime}$.

Taking $\gamma=\alpha$ and $\epsilon=0$ in (7) we get

$$
g^{\prime}\left(\alpha^{\sigma}\right)=c^{-1} g(\alpha)
$$

for any $\alpha$ in $G$. Now taking $\epsilon=-\gamma$ in (7), we have $l_{-\gamma}=l_{\gamma}^{-1}$ for any $\gamma$, and taking $\gamma=2 \zeta$ and $\epsilon=-\zeta$ we have $l_{\zeta}=l_{2 \zeta} l_{-\zeta}$, i.e., $l_{2 \zeta}=l_{\zeta}^{2}$ for any $\zeta$ in $G$. If $f(\alpha, \beta)+g(\alpha-\beta)=0$ then, by (7) and (8), $c f^{\prime}\left(\alpha^{\sigma}, \beta^{\sigma}\right)+g(\alpha-\beta)$ $=0$ and

$$
f^{\prime}\left(\alpha^{\sigma}, \beta^{\sigma}\right)=c^{-1} f(\alpha, \beta) .
$$

Similarly, if $f(\alpha, \beta)-g(\alpha-\beta)=0$ or $4 f(\alpha, \beta)+2 g(\alpha-\beta)=0$ then by taking $\gamma=-\alpha$ and $\epsilon=-\beta$, or $\gamma=2 \alpha$ and $\epsilon=2 \beta$, in (7), we have (9) again. Now suppose that the expressions $f(\alpha, \beta)+g(\alpha-\beta), f(\alpha, \beta)$ $-g(\alpha-\beta)$ and $4 f(\alpha, \beta)+2 g(\alpha-\beta)$ are nonzero. By (7) and (8) we have

$$
l_{-\alpha-\beta}=l_{-\alpha} l_{-\beta}\left[c f^{\prime}\left(\alpha^{\sigma}, \beta^{\sigma}\right)-g(\alpha-\beta)\right][f(\alpha, \beta)-g(\alpha-\beta)]^{-1}
$$

while on the other hand

$l_{-\alpha-\beta}=\left(l_{\alpha+\beta}\right)^{-1}=l_{\alpha}^{-1} l_{\beta}^{-1}\left[c f^{\prime}\left(\alpha^{\sigma}, \beta^{\sigma}\right)+g(\alpha-\beta)\right]^{-1}[f(\alpha, \beta)+g(\alpha-\beta)]$.

Hence $\left[c f^{\prime}\left(\alpha^{\sigma}, \beta^{\sigma}\right)\right]^{2}=[f(\alpha, \beta)]^{2}$. Now similarly by expanding $l_{2 \alpha+2 \beta}$ in two different ways one may see that (9) always holds. It then follows that $l_{\alpha+\beta}=l_{\alpha} l_{\beta}$ for any $\alpha$ and $\beta$ in $G$. We have thus proved one direction of the following theorem. The converse is clear from (7).

Theorem 3. A linear mapping $\sigma: L(G, g, f) \rightarrow L\left(G^{\prime}, g^{\prime}, f^{\prime}\right)$ of one of the simple Lie algebras $L(G, g, f)$ of characteristic zero onto another is an isomorphism if and only if there is an induced isomorphism $\sigma: \alpha \rightarrow \alpha^{\sigma}$ of $G$ onto $G^{\prime}, a$ nonzero scalar $c$ and a homomorphism $l: \alpha \rightarrow l_{\alpha}$ of $G$ into the multiplicative group $F^{*}$ of the base field, such that (6), (8) and (9) hold for all $\alpha$ and $\beta$ in $G$. 
This result in particular applies to automorphisms of $L(G, g, f)$. Thus if $G$ has rank one then the automorphism group of $L(G, g, f)$ is a semidirect product of a normal subgroup $A$ and a subgroup $B$, where $A$ is isomorphic to the group of homomorphisms of $G$ into $F^{*}$ and $B$ is isomorphic to the group of automorphisms of $G$. Also if $G$ is a free abelian group on $n$ generators $\alpha_{1}, \cdots, \alpha_{n}$ and the elements $g\left(\alpha_{i}\right) g\left(\alpha_{j}\right)(1 \leqq i \leqq j \leqq n)$ of $F$ are linearly independent over the rationals, then the automorphism group of $L(G, g, f)$ is again a semidirect product of groups $A$ and $B$, where $A$ is an $n$-fold direct product of $F^{*}$, and $B$ has order 2 or 1 according to whether $f$ vanishes identically or not.

\section{BibliogRAPHY}

1. A. A. Albert, and M. S. Frank, Simple Lie algebras of characteristic p, Univ. e Politec. Torino. Rend. Sem. Mat. vol. 14 (1954-1955) pp. 117-139.

2. Graham Higman, The units of group-rings, Proc. London Math. Soc. vol. 46 (1939-1940) pp. 231-248.

3. S. A. Jennings and Rimhak Ree, On a family of Lie algebras of characteristic $p$, Trans. Amer. Math. Soc. vol. 84 (1957) pp. 192-207.

4. Rimhak Ree, On generalized Witt algebras, Trans. Amer. Math. Soc. vol. 83 (1956) pp. 510-546.

INDIANA UNIVERSITY 